\title{
COMPLICAÇÕES PÓS-OPERATÓRIAS EM TIREOIDECTOMIAS COM OU SEM DRENO
}

\author{
POSTOPERATIVE COMPLICATIONS OF THYROIDECTOMY WITH OR WITHOUT \\ DRAINS
}

\author{
João Gonçalves Filho TCBC-AL ${ }^{1}$; Luiz Paulo Kowalski TCBC-SP²
}

\begin{abstract}
RESUMO: Objetivo: Analisar a ocorrência de complicações pós-operatórias locais (hematoma, seroma e infecção de ferida) e tempo de hospitalização em pacientes submetidos à tireoidectomia com ou sem dreno. Método: Quatrocentos e noventa e oito pacientes submetidos à tireoidectomia foram classificados de acordo com o uso ou não de dreno. Os pacientes foram agrupados para facilitar a descrição em: grupo não drenado; grupo drenado com Penrose e grupo drenado com Hemovac. Cada grupo era composto por 166 pacientes. Resultados: A taxa de complicação foi 7,4\% (37/498). O tempo de hospitalização variou de 1 a 5 dias (mediana, um dia), com $85,6 \%$ dos pacientes no grupo não drenado ficando um dia, enquanto o mesmo ocorreu em $51,8 \%$ dos pacientes do grupo drenado com Hemovac e em apenas 26,9\% dos pacientes do grupo drenado com Penrose. Com isso, o tempo médio de hospitalização foi significativamente maior nos pacientes do grupo drenados com Penrose comparado com os outros grupos $(\mathrm{p}=0,0001)$. Hematoma foi uma complicação encontrada em $1,4 \%$ dos pacientes $(7 / 498)$. Complicações como seroma e infeç̧ão de ferida cirúrgica foram encontradas em $4,4 \%$ e $1,8 \%$ dos pacientes, respectivamente. Nenhuma correlação estatisticamente significativa foi observada quanto à ocorrência de tais complicações e a presença ou não de dreno. Conclusão: O uso rotineiro de dreno em pacientes submetidos à tireoidectomia não apresenta benefícios. A seleção criteriosa dos pacientes e uso de medidas meticulosas de hemostasia são importantes para reduzir o risco de complicações pós-operatórias como hematoma e seroma (Rev. Col. Bras. Cir. 2006; 33(6): 350-353).
\end{abstract}

Descritores: Tireóide; Tireoidectomia; Complicações pós-operatórias; Dreno.

\section{INTRODUÇÃO}

A incidência de nódulo tireoideano palpável é comum na população adulta, variando de 4 a $7 \%{ }^{1}$. A tireoidectomia é indicada no tratamento não somente das lesões malignas, mas também de doenças benignas na presença de sintomas obstrutivos, problemas cosméticos (grandes bócios), hipertireoidismo e na suspeita de associação com doença maligna ${ }^{2,3}$. Desta forma, a tireoidectomia é um procedimento cirúrgico relativamente comum não somente para especialistas em cirurgia de cabeça e pescoço. Os refinamentos técnicos alcançados, particularmente nas últimas décadas, tornaram este procedimento de baixo risco ${ }^{4-6}$.

As principais estruturas sob risco durante a cirurgia da tireóide são os nervos laríngeos recorrentes e as glândulas paratireóides. No entanto, complicações pós-operatórias como hematoma, mesmo sendo uma ocorrência rara nos dias de hoje, quando ocorre pode colocar em risco a vida do paciente. Freqüentemente há necessidade de reintervenção cirúrgica ${ }^{5}$. O uso de dreno nas tireoidectomias tem como objetivos evitar ou diagnosticar precocemente a ocorrência de hematoma, reduzindo o risco para o paciente ${ }^{7-9}$. Nos últimos anos, alguns estudos $^{6,10}$ têm demonstrado que a simples colocação de dreno após tireoidectomia não diminui o risco de hematoma e pode estar associado a um maior risco de infecção de ferida operatória ${ }^{11}$. Além disso, uma série de trabalhos demonstra que drenos selecionados podem não ser usados sem riscos

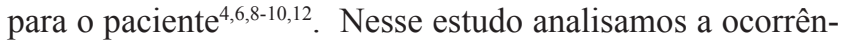
cia de complicações pós-operatórias locais (hematoma, seroma e infecção de ferida) e tempo de hospitalização em pacientes submetidos a tireoidectomia com ou sem o uso de drenos.

\section{MÉTODO}

No período de janeiro de 1990 a dezembro de 2000, 1020 pacientes foram submetidos à tireoidectomias no Departamento de Cirurgia de Cabeça e Pescoço e Otorrinolaringologia e do Centro de Tratamento e Pesquisa Hospital do Câncer A.C. Camargo. Haviam 888 pacientes $(87 \%)$ do sexo feminino e 132 (13\%) do sexo masculino, com idade variando de 2 a 88 anos (mediana, 46 anos). A maior parte destas operações foi realizada por médicos residentes sob a supervisão direta de 10 cirurgiões especialistas em cirurgia de cabeça e pescoço. Em 620 pacientes foi realizada drenagem com hemovac (dreno de sucção); em 224 pacientes utilizou-se dreno de Penrose e em 176 pacientes não foi colocado dreno. Este grupo de pacientes não drenado compreende a base do nosso estudo. $\mathrm{O}$ número de tireoidectomias não drenadas variou durante o período de estudo (Figura 1). A decisão da não colocação de dreno foi tomada ao término da cirurgia após avaliação do espaço morto pós-ressecção, baseado no sangramento transoperatório e nas condições da hemostasia.

1. Departamento de Cirurgia de Cabeça e Pescoço e Otorrinolaringologia do Centro de Tratamento e Pesquisa Hospital do Câncer AC. Camargo.

Recebido em 09/06/06

Aceito para publicação em 10/07/06

Conflito de interesses: nenhum

Fonte de financiamento: nenhuma

Trabalho realizado Departamento de Cirurgia de Cabeça e Pescoço e Otorrinolaringologia do Centro de Tratamento e Pesquisa Hospital do Câncer AC. Camargo. 


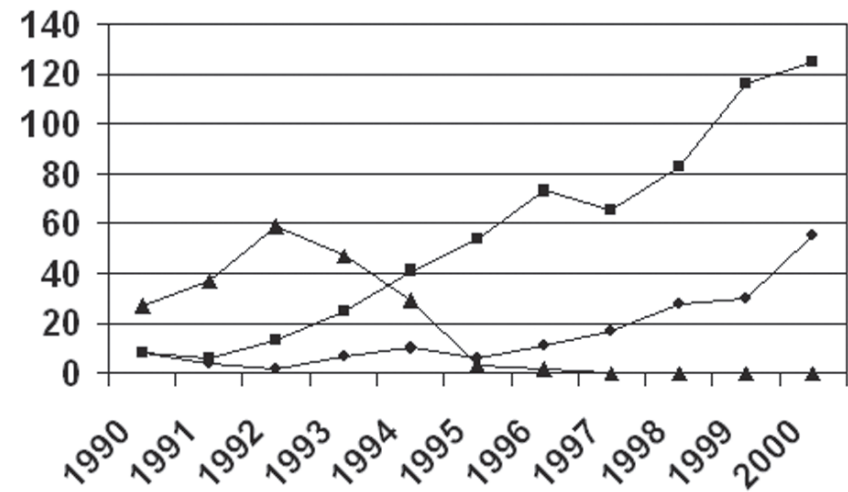

Figura 1 - O número de tireoidectomias por ano de acordo com uso de drenos. Legenda: •, grupo não drenado; $\mathbf{\Delta}$, grupo drenado com Penrose; $\mathbf{\square}$, grupo drenado com Hemovac

Os pacientes foram selecionados de acordo com o tipo de tireoidectomia para constituir grupos de pacientes que se assemelhassem, com o objetivo de facilitar a comparação entre os grupos. Com isso, 166 pacientes de cada grupo foram selecionados. Os pacientes foram agrupados para facilitar a descrição em: grupo não drenado; grupo de pacientes drenados com Penrose e grupo de pacientes drenados com Hemovac. A Tabela 1 apresenta as características dos pacientes incluídos em cada grupo.

A associação entre as variáveis estudadas com a ocorrência de complicações foi avaliada pelo teste do quiquadrado ou teste exato de Fisher quando aplicável. ANOVA foi utilizada para avaliar a diferença entre as médias. Valores de $\mathrm{p}<0,05$ foram considerados significativos. O programa SPSS 12.0 foi usado para análise estatística.

\section{RESULTADOS}

As complicações pós-operatórias ocorreram em 37 dos 498 pacientes estudados (7,4\%), o tempo de hospitalização variou de 1 a 5 dias (mediana, um dia). As complicações ocorreram em 11 pacientes $(6,6 \%)$ do grupo não drenado, em 10 pacientes $(6,0 \%)$ do grupo drenado com Penrose e em 16 pacientes $(9,3 \%)$ do grupo drenado com Hemovac. Não houve diferenças estatisticamente significativas entre a taxa de complicações pós-operatórias e o uso ou não de dreno $(\mathrm{p}=0,405)$. O tempo de hospitalização entre os grupos foi: grupo não drenado, 1 a 3 dias (mediana, um dia); grupo drenado com Penrose, 1 a 5 dias (mediana, dois dias) e grupo drenado com Hemovac, 1 a 4 dias (mediana, um dia). Embora o tempo mediano de hospitalização foi basicamente similar entre os grupos:

Tabela 1 - Características dos grupos estudados.

\begin{tabular}{|c|c|c|c|}
\hline Variável & $\begin{array}{c}\text { Grupo } \\
\text { Sem dreno } \\
\end{array}$ & $\begin{array}{c}\text { Grupo } \\
\text { Penrose } \\
\end{array}$ & $\begin{array}{c}\text { Grupo } \\
\text { Hemovac }\end{array}$ \\
\hline \multicolumn{4}{|l|}{ Sexo } \\
\hline$-M: F$ & $12: 154$ & $23: 143$ & $23: 143$ \\
\hline Idade em anos (mediana) & $13-78 \quad(42)$ & $14-79$ (46) & $8-85(46)$ \\
\hline Nódulo em cm (mediana) & $1-\quad 7 \quad(3)$ & $1-10$ & $1-11$ \\
\hline \multicolumn{4}{|l|}{ Tireoidectomia } \\
\hline - Lobect / Lobec+ ist & 144 & 144 & 144 \\
\hline - Totalização & 7 & 7 & 7 \\
\hline Subtotal & 5 & 5 & 5 \\
\hline
\end{tabular}

Lobect / Lobec+ist: inclui os casos de lobectomia e os casos de lobectomia com istmectomia.

Tabela 2 - Complicação pós-operatória de acordo com o grupo.

\begin{tabular}{lcccc}
\hline Complicação & $\begin{array}{c}\text { Grupo } \\
\text { Sem dreno } \\
\text { N (\%) }\end{array}$ & $\begin{array}{c}\text { Grupo } \\
\text { Penrose } \\
\text { N (\%) }\end{array}$ & $\begin{array}{c}\text { Grupo } \\
\text { Hemovac } \\
\text { N (\%) }\end{array}$ & p \\
\hline Hematoma & $1(0,6)$ & $2(1,2)$ & $4(2,4)$ & 0,363 \\
Seroma & $9(5,4)$ & $6(3,6)$ & $7(4,2)$ & 0,717 \\
Infecção ferida & $2(1,2)$ & $2(1,2)$ & $5(3,0)$ & 0,361 \\
\hline
\end{tabular}

Obs: Um paciente apresentou mais de uma complicação. 
$85,6 \%$ dos pacientes no grupo não drenado ficaram um dia hospitalizados; o mesmo ocorrendo em 51,8\% dos pacientes do grupo drenado com Hemovac e em apenas $26,9 \%$ dos pacientes do grupo drenado com Penrose. Com isso, o tempo médio de hospitalização foi significativamente maior nos pacientes do grupo drenado com Penrose comparado com os outros $\operatorname{grupos}(\mathrm{p}=0,0001)$.

O hematoma foi uma complicação pós-operatória encontrada em apenas $1,4 \%$ dos pacientes (7/498). Somente um paciente no grupo onde não foi utilizado dreno evoluiu com essa complicação. Por outro lado, complicações como seroma e infecção de ferida operatória foram encontradas em $4,4 \%$ e $1,8 \%$ dos pacientes, respectivamente. No entanto, não houve diferença estatisticamente significativa quanto à ocorrência de tais complicações e o uso ou não de dreno (Tabela 2).

\section{DISCUSSÃO}

A tireoidectomia é um procedimento cirúrgico muito comum em nosso meio. São realizadas por cirurgiões com variada formação: cirurgia geral, otorrinolaringologia, cirurgia oncológica e cirurgia de cabeça e pescoço. No princípio da história da cirurgia da glândula tireóide as maiores complicações eram hematoma e infecção. No entanto, com a evolução da prática médica, desde o início do século passado, essas complicações têm se tornado cada vez menos frequentes. A incidência de complicações pós-operatórias como hematoma tem sido relatada na literatura variando de 0 a $4 \%{ }^{8,9,11,12-15}$. O emprego rotineiro de drenos após tireoidectomia com intuito de evitar essa complicação é controverso $0^{4,15,16}$.

Em nosso estudo drenos não foram empregados em 166 casos selecionados de acordo com os critérios do cirurgião. Somente um paciente $(0,6 \%)$ deste grupo evoluiu com hematoma pós-operatório com necessidade de reoperação. Ruark et al. ${ }^{9}$, em uma revisão retrospectiva de 110 operações de tireóide sem o uso de dreno não tiveram nenhum caso de hematoma com necessidade de reoperação. Da mesma forma, Shaha \& Jaffe ${ }^{14} \mathrm{em}$ uma avaliação retrospectiva de 150 pacientes submetidos à tireoidectomia, onde o dreno deixou de ser utilizado de forma seletiva em 115 pacientes, não observaram nenhum caso de hematoma pósoperatório neste grupo de pacientes. Karayacin et al. ${ }^{11} \mathrm{em}$ um extenso estudo retrospectivo com avaliação de 1057 pacientes submetidos a tireoidectomias, sendo 520 drenados e 537 não drenados, observaram que 12 pacientes $(2,3 \%)$ drenados e dois pacientes $(0,37 \%)$ não drenados evoluíram no pós-operatório com hematoma necessitando de reoperação. Recentemente, dois estudos prospectivos randomizados foram conduzidos para avaliar a necessidade do emprego de dreno em tireoidectomia. No primeiro, Khanna et al. ${ }^{17}$ utilizaram-se do emprego da ultrassonografia para avaliar o papel do dreno, em 102 tireoidectomias, de acordo com o volume de sangue coletado no leito tireoideano no $1^{\circ}$ e $7^{\circ}$ dia de pós-operatório, observaram que não houve diferença estatisticamente significativa no volume coletado entre os pacientes que fizeram ou não o uso de dreno, e que o emprego de dreno não apresentou benefício. No outro estudo, Suslu et al. (2006) ${ }^{18}$ estudaram 135 pacientes submetidos à tireoidectomias, sendo 68 drenados e 67 não drenados, notaram que dois pacientes $(2,9 \%)$ drenados apresentaram hematoma necessitando de reoperação e um paciente $(1,5 \%)$ não drenado apresentou hematoma pósoperatório tratado com aspiração, e que o emprego rotineiro de dreno não apresenta nenhum benefício na prevenção de hematoma. Da mesma forma, recente estudos de meta-análises, conduzidos por Corsten et al ${ }^{19}$ e Pothier ${ }^{20}$, baseada em estudos randomizados, avaliaram a efetividade do emprego de dreno em tireoidectomia, observaram que não houve diferença estatística na taxa de hematoma pós-operatório quanto ao uso ou não de dreno. Outros estudos prospectivos randomizados têm demonstrado que o uso rotineiro de dreno após tireoidectomia não apresenta nenhum benefício quanto à prevenção de hematoma pós-operatório quando comparado com pacientes não drenados $6,8,10,12$.

Complicações pós-operatórias como seroma e infecção da ferida operatória têm sido também relacionadas ao uso ou não de drenos. A incidência de infecção da ferida operatória após tireoidectomia varia de 0 a 5\% 9,11,13,17. Em nossa série, a infecção de ferida operatória foi diagnosticada em $1,8 \%$ dos pacientes (9/498). Não observamos correlação estatisticamente significativa quanto à presença ou não de dreno $(\mathrm{p}=0,361)$. Por outro lado, Karayacin et al. ${ }^{11}$ observaram uma taxa significativamente maior de infecção de ferida operatória associada com o uso de dreno. Saslu et al. observaram um aumento do risco de infecção em pacientes drenados quando comparado com pacientes não drenados ${ }^{18}$.

O seroma foi uma complicação pós-operatória encontrada em 22 pacientes $(4,4 \%)$, ocorreu em nove pacientes $(5,4 \%)$ do grupo não drenado, em seis pacientes $(3,6 \%)$ do grupo drenado com Penrose e em sete pacientes $(4,2 \%)$ do grupo drenado com Hemovac. Sendo todos tratados com aspiração. Nenhuma diferença estatisticamente relevante foi evidenciada entre a ocorrência desta complicação e o uso ou não de dreno $(\mathrm{p}=0,717)$. A incidência dessa complicação varia de 0 a $9 \% 0^{9,11,13,17}$.

O tempo de hospitalização tem sido apontado como uma das vantagens do não emprego do dreno após tireoidectomia. Em nossa série, observamos uma significativa diferença entre os grupos com drenos e aquele sem dreno, onde $85,6 \%$ dos pacientes não drenados passaram um dia hospitalizados, enquanto que apenas $26,9 \%$ e $51,8 \%$ dos pacientes drenados com Penrose e dreno de sucção permaneceram hospitalizados o mesmo período $(\mathrm{p}=0,001)$. Do mesmo modo, alguns estudos têm demonstrado um maior período de hospitalização associado com o uso rotineiro de dreno após tireoidectomia ${ }^{12,14}$. Recentemente, Khana et al ${ }^{17}$ e Suslu et $a l .^{18}$ observaram, através de estudos randomizados, que o uso de dreno foi significativamente associado a um maior periodo de permanência hospitalar.

Diante dos resultados, concluímos que o uso rotineiro de dreno em pacientes submetidos à tireoidectomia não apresenta qualquer benefício. A seleção criteriosa dos pacientes e o uso de medidas meticulosas de hemostasia são medidas importantes para evitar complicações pós-operatórias como o hematoma e o seroma. 


\begin{abstract}
Background: This study analyzes the occurrence of local postoperative complications (hematoma, seroma and wound infection) and length of hospital stay in patients submitted to thyroidectomy with or without drains. Methods: Four hundred and ninety eight patients who underwent thyroidectomy were classified into three groups: group not drained; group with Penrose drain; and group with suction drain (Hemovac). Each group was composed by 166 patients. Results: The overall postoperative complication rate was of $7.4 \%$ (37/498). The time of hospitalization varied from 1 to 5 days (median, 1 day), with $85.6 \%$ of the patients in the group not drained staying one day. The same occured in 51.8\% of the patients of the group drained with Hemovac and in only $26.9 \%$ of the patients of the group drained with Penrose. The hospital stay was significantly shorter in the nondrained group compared with the other groups $(p=0.0001)$. Postoperative hematoma was found in 7 patients $(1.4 \%)$. Other local complications as seroma and wound infection occurred in $4.4 \%$ and $1.8 \%$ of the patients, respectively. No significant correlations were observed between these complications and the use or not of drains. Conclusion: The routine use of drains seems to be unnecessary in patients submitted to thyroidectomy. However, the use of meticulous dissection and transoperative hemostasis are important measures to reduce the risk of postoperative complications as hematoma and seroma.
\end{abstract}

Key Words: Thyroid; Thyroidectomy; Postoperative complications; Drain.

\section{REFERÊNCIA}

1. Shah JP. Thyroid and parathyroid. In: Shah JP, editor. Head and neck surgery. $1^{\text {st }}$ ed. New York: Mosby-Wolfe; 1996. p. 393429 .

2. Shindo ML. Considerations in surgery of the thyroid gland. Otolaryngol Clin North Am. 1996;29(4):629-35.

3. Calik A, Kucuktulu U, Cinel A, Bilgin Y, Alhan E, Piskin B. Complications of 867 thyroidectomies performed in a region of endemic goiter in Turkey. Int Surg. 1996;81(3):298-301.

4. Shaha AR, Jaffe BM. Complications of thyroid surgery performed by residents. Surgery. 1988;104(6):1109-14.

5. Shaha AR, Jaffe BM. Practical management of postthyroidectomy hematoma. J Surg Oncol. 1994;57(4):235-8.

6. Debry C, Renou G, Fingerhut A. Drainage after thyroid surgery: a prospective randomized study. J Laryngol Otol. 1999;113(1):4951.

7. Pederson WC, Johnson CL, Gaskill HV 3rd, Aust JB, Cruz AB Jr. Operative management of thyroid disease. Technical considerations in a residency training program. Am J Surg. 1984;148(3):350-2.

8. Wihlborg O, Bergljung L, Martensson H. To drain or not to drain in thyroid surgery. A controlled clinical study. Arch Surg. 1988;123(1):40-1.

9. Ruark DS, Abdel-Misih RZ. Thyroid and parathyroid surgery without drains. Head Neck. 1992;14(4):285-7.

10. Hurtado-Lopez LM, Lopez-Romero S, Rizzo-Fuentes C, Zaldivar-Ramirez FR, Cervantes-Sanchez C. Selective use of drains in thyroid surgery. Head Neck. 2001;23(3):189-93.

11. Karayacin K, Besim H, Ercan F, Hamamci O, Korkmaz A. Thyroidectomy with and without drains. East Afr Med J. 1997;74(7):431-2.

12. Peix JL, Teboul F, Feldman H, Massard JL. Drainage after thyroidectomy: a randomized clinical trial. Int Surg. 1992;77(2):122-4

13. Wax MK, Valiulis AP, Hurst MK. Drains in thyroid and parathyroid surgery. Are they necessary? Arch Otolaryngol Head Neck Surg. 1995;121(9):981-3.
14. Shara AR, Jaffe BM. Selective use of drains in thyroid surgery. J Surg Oncol. 1993;52(4):241-3.

15. Gonçalves Filho J, Kowalski LP. Postoperative complications of thyroidectomy for differentiated thyroid carcinoma. Am J Otolaryngol. 2004;25(4):225-30.

16. Mishra SK, Sharma AK, Thakur S. Outpatient and short-stay thyroid surgery. Head Neck. 1992;14(3):247-8.

17. Khanna J, Mohil RS, Chintamani, Bhatnagar D, Mittal MK, Sahoo M, Mehrotra M. Is the routine drainage after surgery for thyroid necessary? A prospective randomized clinical study. BMC Surg. 2005;5:11.

18. Suslu N, Vural S, Oncel M, Demirca B, Gezen FC, Tuzun B, Erginel T, Dalkilic G. Is the insertion of drains after uncomplicated thyroid surgery always necessary? Surg Today. 2006;36(3):2158.

19. Corsten $\mathrm{M}$, Johnson S, Alherabi A. Is suction drainage an effective means of preventing hematoma in thyroid surgery? A metaanalysis. J Otolaryngol. 2005;34(6):415-7.

20. Pothier DD. The use of drains following thyroid and parathyroid surgery: a meta-analysis. J Laryngol Otol. 2005;119(9):669-71.

Como citar este artigo:

Gonçalves Filho J, Kowalski LP. Complicações pós-operatórias em tireoidectomias com ou sem dreno. Rev Col Bras Cir. [periódico na Internet] 2006 Nov-Dez;33(6). Disponível em URL: www.scielo.br/ rcbc

Endereço para correspondência:

Luiz Paulo Kowalski

Departamento de Cirurgia de Cabeça e Pescoço e Otorrinolaringologia Centro de Tratamento e Pesquisa Hospital do Câncer A. C. Camargo Rua Professor Antonio Prudente, 211- Liberdade

01509-900- São Paulo - SP

Fax: 55-11-3277-6789

E-mail:1p_kowalski@uol.com.br 